\title{
Serum Free Fatty Acids were Associated with Acute Coronary Syndrome and Complexity of Coronary Artery Disease in Premature Coronary Artery Disease Patients
}

\section{Qianglin Guan}

Beijing An Zhen Hospital: Capital Medical University Affiliated Anzhen Hospital

\section{Xiaoteng Ma}

Beijing An Zhen Hospital: Capital Medical University Affiliated Anzhen Hospital

\section{Yan Sun}

Beijing An Zhen Hospital: Capital Medical University Affiliated Anzhen Hospital Hua Shen

Beijing An Zhen Hospital: Capital Medical University Affiliated Anzhen Hospital Jiaming Qiao

Beijing An Zhen Hospital: Capital Medical University Affiliated Anzhen Hospital

Xiaoli Liu

Beijing An Zhen Hospital: Capital Medical University Affiliated Anzhen Hospital

Yujie Zhou ( $\square$ azzyj12@163.com )

Capital Medical University https://orcid.org/0000-0002-9545-1984

\section{Research}

Keywords: Premature coronary artery disease, Free fatty acids, Acute coronary syndrome

Posted Date: October 8th, 2021

DOl: https://doi.org/10.21203/rs.3.rs-955187/v1

License: (9) (i) This work is licensed under a Creative Commons Attribution 4.0 International License. Read Full License 


\section{Abstract}

Background: With the rapid change of lifestyle, the incidence of coronary artery disease (CAD) was gradually rising among young people, but the specific reason for it was unclear. There were many evidences suggesting free fatty acids (FFAs) were involved in progression of CAD, our study was designed to discuss the relationship between serum FFAs and acute coronary syndrome (ACS) and the complexity of coronary artery disease in premature coronary artery disease (pCAD) patients.

Methods: 502 pCAD patients suffering from ACS confirmed by coronary angiography in Beijing Anzhen Hospital from January to December 2019 were enrolled (ACS group), and 141 patients of the same age whose coronary angiography result was negative were selected during the same period (control group). The clinical data and characteristics of CAD among two groups were gathered and compared.

Results: The level of FFAs in ACS group was significantly higher than control group $(P<0.001)$, and the value of FFAs in AMI group was higher than UA $(P<0.05)$ in both genders. The concentration of FFAs in three-vessel disease group was higher than double-vessel disease group and double-vessel disease group was higher than single-vessel disease group. The linear correlation coefficient between FFAs value and SYNTAX score was $0.370, \mathrm{P}<0.001$. The AUC of FFAs to diagnose ACS was $0.798(0.743-0.853, \mathrm{P}<$ $0.001)$ in males, and $0.738(0.677-0.800, P<0.001)$ in females. Logistic regression analysis showed that elevated level of FFAs [odds ratio $(\mathrm{OR})=7.06,95 \%$ confidence interval $(\mathrm{Cl}) 4.40-11.33, \mathrm{P}<0.001$ ] was independently associated with ACS.

Conclusions: Concentration of FFAs is an independent risk factor associated with the incidence of ACS in pCAD patients and positively correlated with the complexity of CAD.

\section{Background}

Cardiovascular disease (CVD) is a major contributor to mortality worldwide[1]. As one of the most widely affected types, coronary artery disease (CAD) refers to a chronic disease caused by various factors, and it leads to blood flow deficiency in the myocardium and myocardial dysfunction, while premature coronary artery disease (pCAD) means that it occurs before the age of 55 in male and before the age of 65 in female[2]. Above half of CVD deaths occurred in Asia among 18.6 million deaths globally in 2019, and approximately $39 \%$ of these were premature[3]. Acute coronary syndrome (ACS) is a sudden and dangerous status clinically, which is the main cause of hospitalizations and deaths in CAD. With the rapid transition of lifestyle, the incidence of ACS among young patients has progressively increased over the years. A cross-sectional study analyzed data of 2 decades from the National Health and Nutrition Examination Surveys (NHANES) in US, it showed men aged 35 to 54 have higher prevalence of MI than women of the same age, and prevalence of $\mathrm{Ml}$ in women had an increasing trend[4]. Moreover, in a large prospective cohort study involving 28778 patients between 1997 and 2008, ST elevation MI (STEMI) was more common in younger patients[5]. The CRAGS study has indicated that prognosis of young CAD patients may be worse because of their aggressive nature, high risk for recurrent coronary events and 
repeated revascularization. It also proposed that specific risk predictors for such patients were unknown yet[6]. The VIRGO study found that only about half of young patients (18-55 years) knew that they were at risk of CAD, while the proportion of people who were informed that they had CAD risk factors or advised to make a risk modification before the onset of CAD was even lower[7]. Since the pathophysiological process of atherosclerotic plaque formation in young ACS patients was distinct from older patients, further exploration of the risk factors of young ACS patients is needed[8].

Free fatty acids (FFAs) are the products of lipid metabolism and the forms of lipid transport in the body[9]. FFAs participate in the synthesis of various lipids and supply energy to myocardium under physiological conditions. Many previous studies have shown that elevated FFAs can damage vascular endothelium, activate inflammatory cells, induce oxidative stress and promote the formation of lipid plaques, which are strong inducers of atherosclerosis[10-15]. However, the relationship between FFAs and PCAD has not been discussed in detail, hence the objective of our study is to ascertain the correlation between FFAs and ACS in PCAD and the complexity of CAD, and confirm whether FFAs could serve as new indictors for earlier clinical identification and diagnosis of ACS.

\section{Methods}

\section{Study population}

Our study retrospectively enrolled males younger than 55 years old and females younger than 65 years old who received coronary angiography in Beijing Anzhen Hospital from January to December 2019. Patients diagnosed with ACS through criteria established by the European Society of Cardiology guidelines were selected to ACS group[16], and individuals with negative coronary angiography were selected to control group. Patients with prior PCl treatment, infectious diseases, malignant tumors, hepatic or renal dysfunction, autoimmune diseases, severe hematological diseases or incomplete clinical data were excluded from this study. Ultimately, 502 patients and 141 subjects were enrolled as ACS and control group, respectively.

\section{Data collection}

Gender, age, height, weight, and clinical history of all patients (history of diabetes, hypertension, hyperlipidemia, smoking, drinking) were recorded. BMI $\left\{\right.$ weight $\left.(\mathrm{kg}) /[\text { height }(\mathrm{m})]^{2}\right\} \geq 28$ was defined as obesity.

Venous blood of all patients was collected after fasting for at least $8 \mathrm{~h}$ and carried to testing laboratory immediately. The automatic analysis method was used to detect total cholesterol (TC), triglyceride (TG), high-density lipoprotein cholesterol (HDL-C), fasting blood glucose (FBG), low-density lipoprotein cholesterol (LDL-C), glycosylated hemoglobin (HbA1c), uric acid, homocysteine (Hcy), high-sensitivity Creactive protein (hsCRP), FFAs, small and dense low-density lipoprotein cholesterol (sdLDL-C), lipoprotein (a) [LP(a)], thyroid stimulating hormone (TSH), complement C1q, hemoglobin and other laboratory indices. 
Coronary angiography results of all subjects were analyzed detailedly, and the location of the lesions and the degree of stenosis were judged visually by two experienced interventional cardiologists. In case of dispute, the third senior physician determined the results. Each coronary artery was evaluated by at least two different positions, and the stenosis of epicardial coronary artery $\geq 50 \%$ of the lumen diameter was defined as coronary artery lesion. On the basis of the involved amount of LAD, LCX and RCA lesions, individuals of ACS group were divided into three-vessel disease, double-vessel and single-vessel group, and involvement of the left main (LM) was treated as LAD and LCX, and classified as double-vessel disease. The SYNTAX score was obtained by synthetically analyzing coronary angiography results, which was segmented and weighted according to dominant types of coronary arteries and involved vessels by integrating degree of stenosis, lesion time, length, bifurcation lesions, tortuosity, calcification, thrombosis and other factors[17].

\section{Statistical analysis}

Data were statistically analyzed and processed by software SPSS version 19.0. Mean \pm standard deviation (SD) or median (25th and 75th percentiles) was used to present continuous data. Two groups of continuous data were compared by utilizing Student's or Mann-Whitney U test. One-way ANOVA or nonparametric test was applied to calculate the differences between multiple groups. Classified variables were are displayed in the form of counts (percentages), and chi-square test was utilized to reflect differences. The Fisher's exact test was employed when the data did not meet the chi-square test conditions. Spearman correlation analysis was applied to judge relationship between FFAs value and SYNTAX score. ROC curve for FFAs to diagnose ACS was drawn, and AUC and optimal cut-off value were calculated. Multivariate logistic regression analysis was carried out to probe the independent influence of FFAs on ACS and calculate odds ratio (OR). Taking bilateral $P$ value $<0.05$ as statistically significance.

\section{Results}

\section{Baseline characteristics}

A total of 643 subjects (age $49.3 \pm 8.2$ years; $61.0 \%$ men) were incorporated in our study, consisting of 502 individuals in ACS group (age $48.6 \pm 8.2$ years; $66.5 \%$ men) and 141 individuals in control group (age $51.7 \pm 7.8$ years; $41.4 \%$ men). The average age of men was $44.9 \pm 6.2$ years old, and women was $56.1 \pm$ 6.0 years old. Among all patients, $33.6 \%$ were obese, $29.2 \%$ with diabetes, $57.9 \%$ with hypertension, $53.7 \%$ with hyperlipidemia, $44.8 \%$ had a history of smoking, and $27.7 \%$ had a history of drinking. Patients of ACS group ( $48.6 \pm 8.2$ years) were significantly younger than control group (51.7 \pm 7.8 years). ACS group patients had significantly higher proportion in male, obesity, diabetes, high blood pressure, smoking than control group. The level of TG, FBG, HbA1c, hsCRP, uric acid, Hcy, FFAs, LP(a) and hemoglobin in ACS group patients was significantly higher than control group. On the contrary, control group patients had significantly higher level of TC, LDL-C and HDL-C. More detailed baseline characteristics were demonstrated in Table 1. 
Table 1

Baseline characteristics of the study population

\begin{tabular}{|c|c|c|c|c|}
\hline & Total $(n=643)$ & $\begin{array}{l}\text { ACS group }(n= \\
502)\end{array}$ & $\begin{array}{l}\text { Control group }(\mathrm{n}= \\
141)\end{array}$ & $\begin{array}{l}P \\
\text { value }\end{array}$ \\
\hline Age, years & $49.3 \pm 8.2$ & $48.6 \pm 8.2$ & $51.7 \pm 7.8$ & $<0.001$ \\
\hline Sex, male, $\mathrm{n}(\%)$ & $392(61.0)$ & $334(66.5)$ & $58(41.1)$ & $<0.001$ \\
\hline Obesity, n (\%) & 216(33.6) & 186(37.1) & $30(21.3)$ & $<0.001$ \\
\hline Hyperlipidemia, n (\%) & $345(53.7)$ & $280(55.8)$ & $65(46.1)$ & 0.042 \\
\hline $\begin{array}{l}\text { Diabetes mellitus, } \mathrm{n} \\
(\%)\end{array}$ & 188(29.2) & 166(33.1) & $22(16.5)$ & $<0.001$ \\
\hline Hypertension, n (\%) & $372(57.9)$ & $306(61.0)$ & $66(46.8)$ & 0.003 \\
\hline Smoking, n (\%) & $288(44.8)$ & $239(47.6)$ & $49(34.8)$ & 0.007 \\
\hline drinking, n (\%) & 178(27.7) & $143(28.5)$ & $35(24.8)$ & 0.390 \\
\hline TG, mmol/L & $1.98 \pm 1.79$ & $2.07 \pm 1.95$ & $1.65 \pm 0.93$ & $<0.001$ \\
\hline $\mathrm{TC}, \mathrm{mmol} / \mathrm{L}$ & $4.33 \pm 1.14$ & $4.26 \pm 1.14$ & $4.54 \pm 1.12$ & 0.012 \\
\hline $\mathrm{LDL}, \mathrm{mmol} / \mathrm{L}$ & $2.59 \pm 0.96$ & $2.54 \pm 0.96$ & $2.75 \pm 0.93$ & 0.025 \\
\hline $\mathrm{HDL}, \mathrm{mmol} / \mathrm{L}$ & $1.06 \pm 0.28$ & $1.02 \pm 0.26$ & $1.19 \pm 0.28$ & $<0.001$ \\
\hline FBG, mmol/L & $6.21 \pm 2.14$ & $6.36 \pm 2.26$ & $5.67 \pm 1.53$ & $<0.001$ \\
\hline $\mathrm{HbA} 1 \mathrm{c}, \%$ & $6.28 \pm 1.25$ & $6.40 \pm 1.34$ & $5.87 \pm 0.75$ & $<0.001$ \\
\hline hsCRP, mg/L & $\begin{array}{l}1.40(0.60 \\
4.00)\end{array}$ & $1.80(0.70,4.80)$ & $0.90(0.50,2.40)$ & $<0.001$ \\
\hline Uric acid, $\mu \mathrm{mol} / \mathrm{L}$ & $358.54 \pm 95.33$ & $367.46 \pm 96.00$ & $326.74 \pm 85.98$ & $<0.001$ \\
\hline $\mathrm{Hcy}, \mu \mathrm{mol} / \mathrm{L}$ & $14.74 \pm 8.60$ & $15.24 \pm 9.08$ & $12.94 \pm 6.33$ & 0.001 \\
\hline FFAs, mmol/L & $0.55 \pm 0.23$ & $0.59 \pm 0.23$ & $0.42 \pm 0.15$ & $<0.001$ \\
\hline $\mathrm{LP}(\mathrm{a}), \mathrm{mmol} / \mathrm{L}$ & $\begin{array}{l}0.10(0.04 \\
0.28)\end{array}$ & $0.10(0.03,0.32)$ & $0.07(0.04,0.20)$ & 0.003 \\
\hline sdLDL-C, mmol/L & $0.81 \pm 0.38$ & $0.81 \pm 0.38$ & $0.79 \pm 0.37$ & 0.636 \\
\hline TSH, mIU/L & $2.05 \pm 1.52$ & $2.01 \pm 1.54$ & $2.20 \pm 1.46$ & 0.184 \\
\hline $\mathrm{C} 1 \mathrm{q}, \mathrm{mg} / \mathrm{L}$ & $187.84 \pm 31.78$ & $188.19 \pm 32.02$ & $186.60 \pm 30.98$ & 0.600 \\
\hline Hemoglobin, $\mathrm{g} / \mathrm{L}$ & $145.87 \pm 17.19$ & $147.01 \pm 16.68$ & $141.80 \pm 18.42$ & 0.001 \\
\hline
\end{tabular}

\section{Comparison of FFAs value between groups}


The level of FFAs in the AMI group (0.63 \pm 0.32$)$ was evidently higher than unstable angina (UA) group $(0.54 \pm 0.18)$, and that in UA group was significantly higher than control group $(0.37 \pm 0.12)$, and similar results were found in the female patients $(0.77 \pm 0.31$ vs $0.62 \pm 0.20$ vs $0.46 \pm 0.16)$ (Table 2, Figure 1$)$. The FFAs value of the double-vessel group $(0.59 \pm 0.31)$ was significantly higher than single-vessel group $(0.48 \pm 0.16)$ among male patients, while the difference between three-vessel group and double-vessel group was also statistically significant $(0.62 \pm 0.16$ vs $0.59 \pm 0.31, P<0.05)$. Among female patients, the FFAs level of the three-vessel group was significantly higher than that of the double-vessel group, and the double-vessel group was significantly higher than the single-vessel group ( $0.75 \pm 0.18$ vs $0.66 \pm 0.22$ vs $0.53 \pm 0.22)$. The SYNTAX scores between the three groups of in both genders were significantly different $(P<0.05$ between any two groups) (Table 3 , Figure 2 ).

Table 2

Comparison of FFAs level in different CAD types

\begin{tabular}{|lllll|}
\hline & Control group & UA group & AMl group & P value \\
\hline Male, $\mathrm{n}$ & 58 & 243 & 91 & \\
\hline FFAs, $\mathrm{mmol} / \mathrm{L}$ & $0.37 \pm 0.12$ & $0.54 \pm 0.18^{\dagger}$ & $0.63 \pm 0.32^{\dagger, \ddagger}$ & $<0.001$ \\
\hline Female, $\mathrm{n}$ & 83 & 143 & 25 & \\
\hline FFAs, $\mathrm{mmol} / \mathrm{L}$ & $0.46 \pm 0.16$ & $0.62 \pm 0.20^{\dagger}$ & $0.77 \pm 0.31^{\dagger, \ddagger}$ & $<0.001$ \\
\hline${ }^{\dagger} \mathrm{P}<0.05$ vs Control group. & & & \\
\hline${ }^{\ddagger} \mathrm{P}<0.05$ vs UA group. & & & \\
\hline
\end{tabular}

Table 3

Comparison of FFAs level in various degrees of CAD complexity

\begin{tabular}{|c|c|c|c|c|}
\hline & Single-vessel disease & Double-vessel disease & Three-vessel disease & P value \\
\hline Male, $\mathrm{n}$ & 119 & 117 & 98 & \\
\hline FFAs, mmol/L & $0.48 \pm 0.16$ & $0.59 \pm 0.31^{\rrbracket}$ & $0.62 \pm 0.16^{\square, \bigotimes}$ & $<0.001$ \\
\hline SYNTAX score & $8.9 \pm 5.6$ & $15.7 \pm 8.5^{\rrbracket}$ & 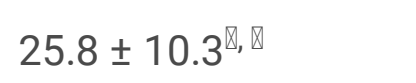 & $<0.001$ \\
\hline Female, $\mathrm{n}$ & 64 & 56 & 48 & \\
\hline FFAs, $\mathrm{mmol} / \mathrm{L}$ & $0.53 \pm 0.22$ & $0.66 \pm 0.22^{\rrbracket}$ & $0.75 \pm 0.18^{\bigotimes, \bigotimes}$ & $<0.001$ \\
\hline SYNTAX score & $8.1 \pm 5.8$ & $16.2 \pm 9.3^{\rrbracket}$ & $26.4 \pm 10.6^{\llbracket, \bigotimes}$ & $<0.001$ \\
\hline \multicolumn{5}{|c|}{${ }^{{ }} \mathrm{P}<0.05$ vs Single-vessel disease. } \\
\hline$<0.05$ vs Do & essel disease. & & & \\
\hline
\end{tabular}




\section{Correlation between FFAs level and varying variables}

Spearman correlation analysis was applied to inquiry relationship between level of FFAs and different continuous variables in this study, which revealed FFAs level was positively correlated with FBG $(r=$ $0.201, P<0.001), \operatorname{HbA} 1 \mathrm{c}(r=0.166, \mathrm{P}<0.001)$, SYNTAX score $(r=0.370, P<0.001)$ (Figure 3$)$ and not related to other factors (all $P>0.05$ ). As listed in Table 4.

Table 4

correlation coefficients between FFAs level and varying variables

\begin{tabular}{|lll|}
\hline Variables & $\mathbf{r}$ & P value \\
\hline Age & 0.067 & 0.092 \\
\hline BMI & 0.046 & 0.253 \\
\hline TG & 0.009 & 0.820 \\
\hline TC & 0.062 & 0.118 \\
\hline LDL-C & 0.005 & 0.901 \\
\hline HDL-C & 0.042 & 0.292 \\
\hline FBG & 0.201 & $<0.001$ \\
\hline HbA1c & 0.166 & $<0.001$ \\
\hline hsCRP & 0.073 & 0.064 \\
\hline Uric acid & -0.026 & 0.505 \\
\hline Hcy & -0.014 & 0.722 \\
\hline LP(a) & 0.068 & 0.084 \\
\hline sdLDL-C & 0.013 & 0.735 \\
\hline SYNTAX score & 0.370 & $<0.001$ \\
\hline
\end{tabular}

\section{Diagnostic value of FFAs for ACS in different genders}

The ROC curve for diagnosing ACS was plotted in Figure 4. The area under the curve (AUC) of FFAs in males for the diagnosis of ACS was 0.798 (95\% Cl: 0.743-0.853), and the optimal cut-off value was $0.475 \mathrm{mmol} / \mathrm{L}$, of which the sensitivity was $65.6 \%$, the specificity was $82.8 \%(P<0.001)$, and the Youden index was 0.483 . The AUC of FFAs in female patients to diagnose ACS was 0.738 (95\% Cl: $0.677-0.800$ ), the optimal cut-off value was $0.565 \mathrm{mmol} / \mathrm{L}$, of which the sensitivity was $64.9 \%$, the specificity was $77.1 \%(P<0.001)$, and the Youden index was 0.420 . Furthermore, diagnostic capacity of FFAs was notably greater than other indicators in both males and females. 


\section{Multivariate logistic regression analysis}

Gender, obesity, hyperlipidemia, diabetes, hypertension, history of smoking, history of drinking, and FFAs elevation based on optimal cut-off value (male $\geq 0.475 \mathrm{mmol} / \mathrm{L}$, female $\geq 0.565 \mathrm{mmol} / \mathrm{L}$ ) were included in this multivariate logistic regression analysis (Table 5, Figure 5 ). The analysis presented that male, diabetes, hypertension, and increased FFAs level were independently associated with the occurrence of ACS (all $\mathrm{P}<0.05)$, and Elevated level of FFAs was the strongest risk factor $(\mathrm{OR}=7.06 ; 95 \% \mathrm{Cl}$ : $4.40-11.33, P<0.001)$.

Table 5

Multivariate logistic regression analysis of risk factors for ACS

\begin{tabular}{|llll|}
\hline Variables & OR & $95 \% \mathrm{Cl}$ & P value \\
\hline Male & 3.61 & $1.98-6.59$ & $<0.001$ \\
\hline Obesity & 1.45 & $0.88-2.39$ & 0.141 \\
\hline Hyperlipidemia & 1.29 & $0.84-1.98$ & 0.249 \\
\hline Diabetes mellitus & 2.11 & $1.22-3.65$ & 0.007 \\
\hline Hypertension & 1.68 & $1.09-2.61$ & 0.020 \\
\hline Smoking & 1.02 & $0.54-1.92$ & 0.949 \\
\hline Drinking & 0.58 & $0.31-1.08$ & 0.084 \\
\hline Elevated level of FFAs & 7.06 & $4.40-11.33$ & $<0.001$ \\
\hline
\end{tabular}

\section{Discussion}

Fatty acids are important organic acids in the human body, which are inseparable from the metabolism of various lipids. They are the main raw materials for the synthesis of triglycerides, phospholipids and cholesterols, as well as essential substances to supply energy[18]. FFAs are the type of fatty acids not covalently bound to other substances in the blood, which are also named as non-esterified fatty acids (NEFAs), mostly derived from lipid metabolism and decomposition[9]. As early as the last century, a study has found that high concentrations of FFAs can increase myocardial oxygen consumption in rats, which can further damage ischemic myocardium and reduce the contractility of hypoxic myocardium[19]. For the past few years, more studies have shown that FFAs are closely bound up with CVD. Oliver et al. observed that FFAs increased significantly in patients with AMI, and elevated FFAs can lead to metabolic crisis in the impaired myocardium along with the result of ventricular arrhythmia and an increase in early mortality[20]. Meanwhile a study conducted by Jouven et al. proposed that the concentration of circulating FFAs can be an independent risk factor for cardiac sudden death in middle-aged men[21]. Research by Carlsson et al. has found offspring of parents with CVD history had higher fasting serum FFAs level, which pointed out that elevated serum FFAs concentration was associated with an increased 
risk of familial CVD, and it is speculated that FFA may be involved in the pathological process of atherosclerosis[22].

Our study showed that FFAs significantly increased in ACS with PCAD, and degree of elevation of FFAs level in AMI patients was more remarkable than UA patients. Further subgroup analysis manifested that ACS patients with three-vessel disease had higher FFAs level than patients with double-vessel and singlevessel disease irrespective of the gender. After introducing the SYNTAX score to quantify the complexity of CAD, it was found that the serum FFAs value is linearly positively correlated with the FBG, HbA1C and SYNTAX score, and the maximum correlation coefficient is that between FFAs level and SYNTAX score. Furthermore, FFAs can not only assist in the diagnosis of ACS in PCAD patients, but elevated concentration of FFAs is an independent risk factor for the occurrence of ACS with PCAD, which is more relevant than factors such as male, diabetes mellitus, and hypertension.

As we all know, since the CVD risk factors were proposed in the Framingham Heart Study, risk factors like hypertension, diabetes, obesity, smoking history, dyslipidemia and etc. have been identified by the majority of scholars[23]. Nevertheless, FFAs were proposed by Pilz et al. as new indicators to assess CVD risk factors in the early years. They comprehensively analyzed the role of FFAs in promoting atherosclerosis, and found that FFAs can cause the apoptosis of vascular endothelial cells by activating protein phosphatase $2 \mathrm{C} \beta$ (PP2C $\beta$ ), and high FFAs concentrations can induce expression of endothelial inflammatory factors such as monocyte chemoattractant protein-1 (MCP-1), tumor necrosis factor-alpha (TNF-a), vascular adhesion molecule-1 (VCAM-1), interleukin-8 (IL-8) and cell adhesion molecule-1 (ICAM1) to activate inflammation response, as well as participate in the process of macrophages transforming into foam cells by affecting cholesterol transport[24]. In addition, the study conducted by Westphal et al. in individuals with a low traditional CVD risk elucidated that the level of FFAs in male CAD patients elevated most significantly after applying interventions in lipid metabolism. They postulated that FFAs leads to CAD through three pathways: directly acting on blood vessels or myocardium, stimulating the liver to release atherogenic lipoprotein, inducing intra-plasmatic processes of exchange to produce atherogenic lipoprotein[25].

Due to various mechanisms of FFAs promoting the formation of atherosclerosis, the relationship between FFAs and the complexity of CAD has been disclosed by many scholars. A study from He et al. revealed FFAs value was positively correlated with the Gensini score of CAD in elderly patients $(r=0.394, P=$ 0.005)[26]. Whereas after replacing the Gensini score with the SYNTAX score in this study, which is more widely used in clinical practice, it was found similar relationship exists between FFA and SYNTAX score in younger ACS patients, indicating that the FFAs value can indeed reflect the complexity of coronary artery disease. Not only that, our study demonstrated the association between the level of FFAs and the types of ACS at the same time, and FFAs level in AMI was significantly higher than that in UA and the control group, indicating that FFAs also reflect the degree of danger of ACS to some extent. However, this study did not use quantitative tools to stratify ACS risk, which requires further research and exploration in the future. In terms of the diagnostic value of FFA, Kan et al. recently drew the ROC curve of FFAs for the diagnosis of AMI and found that its AUC was 0.77 in men and 0.80 in women, which was greater than 
hsCRP and ProBNP[27]. Our study expanded the application of FFAs to ACS patients and found that it is equally feasible in assisting the diagnosis of ACS in patients with PCAD. Many scholars have explored the risk factors of pCAD previously[28-31], but there has never been a study discussing the impact of FFAs on it. The correlation between FFAs and the occurrence of ACS in PCAD patients is analyzed for the first time in our study, and the results prove that elevated value of FFAs is a strong independent risk factor affecting its progress.

\section{Limitations}

There are actually some limitations to our study: (1) Our study is a single-center retrospective study, which will inevitably lead to deviations in selection of patients. (2) The subjects of our study are all hospitalized patients undergoing coronary angiography who have higher CVD risk factors, and further studies based on the community and the general population are necessary to be implemented to explore relationship between FFAs and PCAD. (3) The components of FFAs are complex, including oleic acid, linoleic acid, stearic acid, etc. It is unclear which component affects PCAD, and the specific effects of different components on PCAD are expected to be further researched in the future. (4) We only discovered the numerical correlation between FFAs and PCAD, and the deeper connection between FFAs and PCAD, such as genetic aspects, requires subsequent research to clarify.

\section{Conclusions}

Concentration of FFAs is an independent risk factor associated with the incidence of ACS in PCAD patients and positively correlated with the complexity of CAD. The level of FFAs can assist in the diagnosis of ACS and predict the complexity of their coronary artery disease in PCAD individuals. The higher the level of FFAs, the more likely it is to be diagnosed as ACS, and the lesions of CAD are more complicated. FFAs can be acted as a new biomarker for clinical prediction of pCAD, helping to identify population at high risk of CAD earlier.

\section{Abbreviations}

CAD

Coronary artery disease

FFAs

Free fatty acids

ACS

Acute coronary syndrome

PCAD

premature Coronary artery disease

AMI

Acute myocardial infarction

UA 
Unstable angina

ROC

Receiver-operating characteristic

AUC

Area under the curve

OR

odds ratio

CVD

Cardiovascular disease

STEMI

ST elevation myocardial infarction

BMI

Body mass index

TG

Triglyceride

TC

Total cholesterol

LDL-C

Low-density lipoprotein cholesterol

HDL-C

High-density lipoprotein cholesterol

FBG

Fasting blood glucose

hsCRP

High-sensitivity C-reactive protein

Hcy

Homocysteine

LP(a)

Lipoprotein (a)

sdLDL-C

Small and dense low-density lipoprotein cholesterol

$\mathrm{TSH}$

Thyroid stimulating hormone

LAD

Left anterior descending artery

LCX

Left circumflex artery

RCA

Right coronary artery

LM 
Left main

SD

Standard deviation

NEFAs

Non-esterified fatty acids

ProBNP

Pro-Brain natriuretic peptide.

\section{Declarations}

\section{Acknowledgements}

Not applicable.

\section{Authors' contributions}

QLG contributed substantially to data acquisition, data processing, data analysis and manuscript writing. XTM and HS corrected the manuscript and gave statistical support. YS and JMQ collected and checked partial data. XLL and YJZ designed this study and offered academic assistance. All authors read and approved the final manuscript.

\section{Funding}

Our study was supported by grants from the National Key Research and Development Program of China (2017YFC0908800), Beijing Municipal Administration of Hospitals' Mission plan (SML20180601), Beijing Municipal Health Commission: Jing 19-15, Project funded by China Postdoctoral Science Foundation (2021M692253)

\section{Availability of data and materials}

The datasets used and/or analyzed during the current study are available from the corresponding author on reasonable request.

\section{Ethics approval and consent to participate}

In view of the retrospective nature of this study, the requirement for informed consent was waived. The research protocol was approved by the Clinical Research Ethics Committee of Beijing Anzhen Hospital, Capital Medical University.

\section{Consent for publication}

Not applicable.

\section{Competing interests}


The authors declare that they have no competing interests.

\section{References}

1. Global, regional, and national age-sex-specific mortality for 282 causes of death in 195 countries and territories, 1980-2017: a systematic analysis for the Global Burden of Disease Study 2017. Lancet 2018, 392:1736-1788.

2. Arnett DK, Blumenthal RS, Albert MA, Buroker AB, Goldberger ZD, Hahn EJ, Himmelfarb CD, Khera A, Lloyd-Jones D, McEvoy JW, et al. 2019 ACC/AHA Guideline on the Primary Prevention of Cardiovascular Disease: Executive Summary: A Report of the American College of Cardiology/American Heart Association Task Force on Clinical Practice Guidelines. Circulation. 2019;140:e563-95.

3. Zhao D. Epidemiological Features of Cardiovascular Disease in Asia. JACC: Asia. 2021;1:1-13.

4. Towfighi A, Zheng L, Ovbiagele B. Sex-specific trends in midlife coronary heart disease risk and prevalence. Arch Intern Med. 2009;169:1762-6.

5. Schoenenberger AW, Radovanovic D, Stauffer JC, Windecker S, Urban P, Niedermaier G, Keller PF, Gutzwiller F, Erne P. Acute coronary syndromes in young patients: presentation, treatment and outcome. Int J Cardiol. 2011;148:300-4.

6. Lautamäki A, Airaksinen KE, Kiviniemi T, Vinco G, Ribichini F, Gunn J, Anttila V, Heikkinen J, Korpilahti $\mathrm{K}$, Karjalainen $\mathrm{P}$, et al. Prognosis and disease progression in patients under 50 years old undergoing PCI: the CRAGS (Coronary aRtery diseAse in younG adultS) study. Atherosclerosis. 2014;235:483-7.

7. Leifheit-Limson EC, D'Onofrio G, Daneshvar M, Geda M, Bueno H, Spertus JA, Krumholz HM, Lichtman JH. Sex Differences in Cardiac Risk Factors, Perceived Risk, and Health Care Provider Discussion of Risk and Risk Modification Among Young Patients With Acute Myocardial Infarction: The VIRGO Study. J Am Coll Cardiol. 2015;66:1949-57.

8. Barbero U, Scacciatella P, lannaccone M, D'Ascenzo F, Niccoli G, Colombo F, Ugo F, Colangelo S, Mancone $\mathrm{M}$, Calcagno $\mathrm{S}$, et al. Culprit plaque characteristics in younger versus older patients with acute coronary syndromes: An optical coherence tomography study from the FORMIDABLE registry. Catheter Cardiovasc Interv. 2018;92:E1-8.

9. Koutsari C, Jensen MD. Thematic review series: patient-oriented research. Free fatty acid metabolism in human obesity. J Lipid Res. 2006;47:1643-50.

10. Tripathy D, Mohanty P, Dhindsa S, Syed T, Ghanim H, Aljada A, Dandona P. Elevation of free fatty acids induces inflammation and impairs vascular reactivity in healthy subjects. Diabetes. 2003;52:2882-7.

11. de Jongh RT, Serné EH, ljzerman RG, de Vries G, Stehouwer CD. Free fatty acid levels modulate microvascular function: relevance for obesity-associated insulin resistance, hypertension, and microangiopathy. Diabetes. 2004;53:2873-82. 
12. Oram JF, Bornfeldt KE. Direct effects of long-chain non-esterified fatty acids on vascular cells and their relevance to macrovascular complications of diabetes. Front Biosci. 2004;9:1240-53.

13. Hufnagel B, Dworak M, Soufi M, Mester Z, Zhu Y, Schaefer JR, Klumpp S, Krieglstein J. Unsaturated fatty acids isolated from human lipoproteins activate protein phosphatase type 2Cbeta and induce apoptosis in endothelial cells. Atherosclerosis. 2005;180:245-54.

14. Lloyd EE, Gaubatz JW, Burns AR, Pownall HJ. Sustained elevations in NEFA induce cyclooxygenase-2 activity and potentiate THP-1 macrophage foam cell formation. Atherosclerosis. 2007;192:49-55.

15. Azekoshi Y, Yasu T, Watanabe S, Tagawa T, Abe S, Yamakawa K, Uehara Y, Momomura S, Urata H, Ueda S. Free fatty acid causes leukocyte activation and resultant endothelial dysfunction through enhanced angiotensin II production in mononuclear and polymorphonuclear cells. Hypertension. 2010;56:136-42.

16. Collet JP, Thiele H, Barbato E, Barthélémy O, Bauersachs J, Bhatt DL, Dendale P, Dorobantu M, Edvardsen T, Folliguet T, et al. 2020 ESC Guidelines for the management of acute coronary syndromes in patients presenting without persistent ST-segment elevation. Eur Heart J. 2021;42:1289-367.

17. Sianos G, Morel MA, Kappetein AP, Morice MC, Colombo A, Dawkins K, van den Brand M, Van Dyck N, Russell ME, Mohr FW, Serruys PW. The SYNTAX Score: an angiographic tool grading the complexity of coronary artery disease. Eurolntervention. 2005;1:219-27.

18. Gurr MI, Harwood JL, Frayn KN: Lipid Biochemistry, 5th Edition. Lipid Biochemistry, 5th Edition; 2008.

19. Henderson AH, Most AS, Parmley WW, Gorlin R, Sonnenblick EH. Depression of myocardial contractility in rats by free fatty acids during hypoxia. Circ Res. 1970;26:439-49.

20. Oliver MF. Fatty acids and the risk of death during acute myocardial ischaemia. Clin Sci (Lond). 2015;128:349-55.

21. Jouven $X$, Charles MA, Desnos $M$, Ducimetière P. Circulating nonesterified fatty acid level as a predictive risk factor for sudden death in the population. Circulation. 2001;104:756-61.

22. Carlsson M, Wessman Y, Almgren P, Groop L. High levels of nonesterified fatty acids are associated with increased familial risk of cardiovascular disease. Arterioscler Thromb Vasc Biol. 2000;20:158894.

23. Andersson C, Johnson AD, Benjamin EJ, Levy D, Vasan RS. 70-year legacy of the Framingham Heart Study. Nat Rev Cardiol. 2019;16:687-98.

24. Pilz S, März W. Free fatty acids as a cardiovascular risk factor. Clin Chem Lab Med. 2008;46:429-34.

25. Westphal S, Gekeler GH, Dierkes J, Wieland H, Luley C. A free fatty acid tolerance test identifies patients with coronary artery disease among individuals with a low conventional coronary risk profile. Heart Vessels. 2002;16:79-85.

26. He LY, Zhao JF, Han JL, Shen SS, Chen XJ. Correlation between serum free fatty acids levels and Gensini score in elderly patients with coronary heart disease. J Geriatr Cardiol. 2014;11:57-62. 
27. Kan Y, Wang H, Lu J, Lin Z, Lin J, Gong P. Significance of plasma free fatty acid level for assessing and diagnosing acute myocardial infarction. Biomark Med. 2020;14:739-47.

28. Hindieh W, Pilote L, Cheema A, Al-Lawati H, Labos C, Dufresne L, Engert JC, Thanassoulis G. Association Between Family History, a Genetic Risk Score, and Severity of Coronary Artery Disease in Patients With Premature Acute Coronary Syndromes. Arterioscler Thromb Vasc Biol. 2016;36:128692.

29. Chieng D, Pang J, Ellis KL, Hillis GS, Watts GF, Schultz CJ. Elevated lipoprotein(a) and low-density lipoprotein cholesterol as predictors of the severity and complexity of angiographic lesions in patients with premature coronary artery disease. J Clin Lipidol. 2018;12:1019-26.

30. Zeitouni M, Clare RM, Chiswell K, Abdulrahim J, Shah N, Pagidipati NP, Shah SH, Roe MT, Patel MR, Jones WS. Risk Factor Burden and Long-Term Prognosis of Patients With Premature Coronary Artery Disease. J Am Heart Assoc. 2020;9:e017712.

31. Kryczka KE, Kruk M, Demkow M, Lubiszewska B: Fibrinogen and a Triad of Thrombosis, Inflammation, and the Renin-Angiotensin System in Premature Coronary Artery Disease in Women: A New Insight into Sex-Related Differences in the Pathogenesis of the Disease. Biomolecules 2021, 11.

\section{Figures}




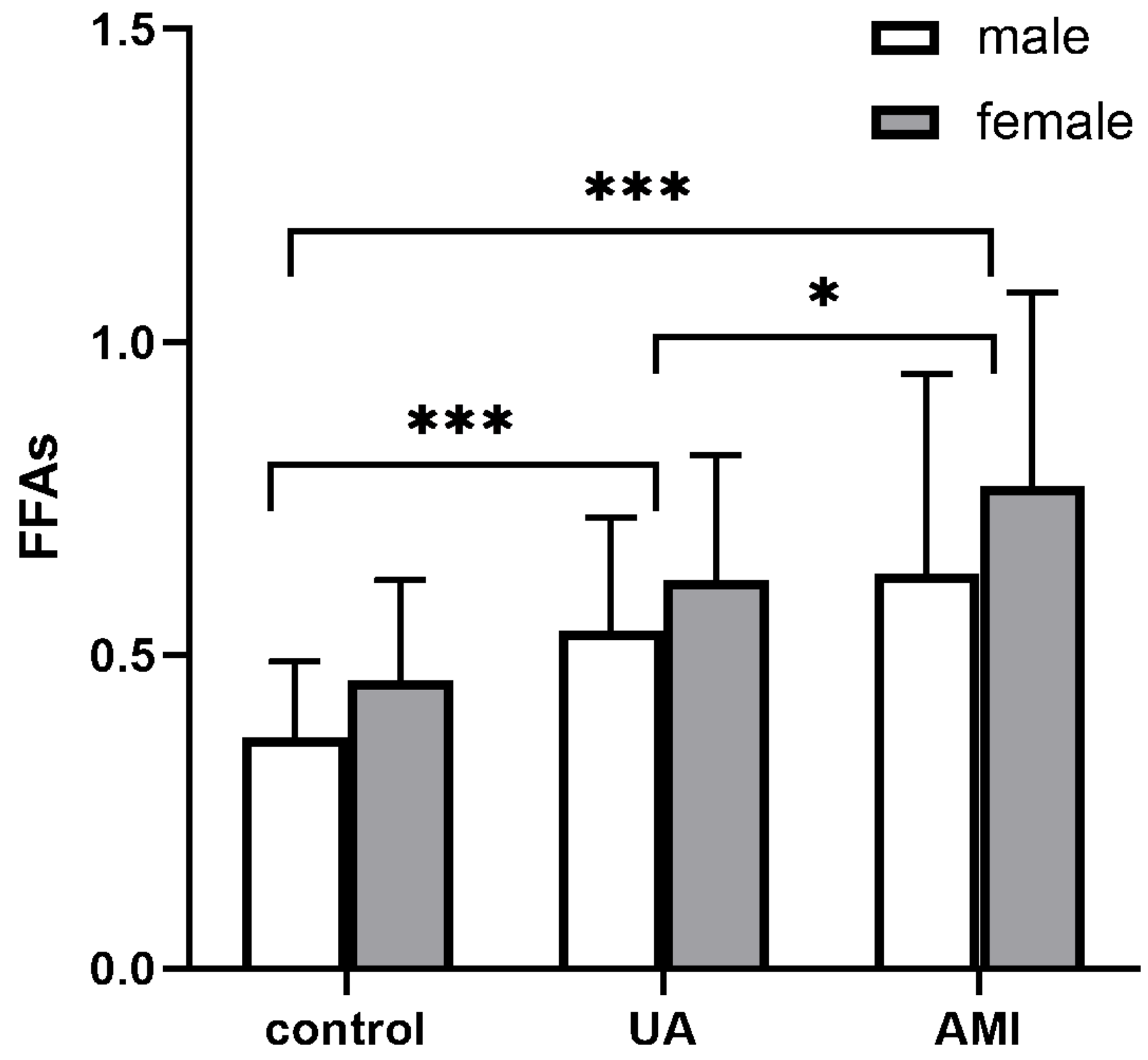

Figure 1

FFAs level in different genders based on CAD types 


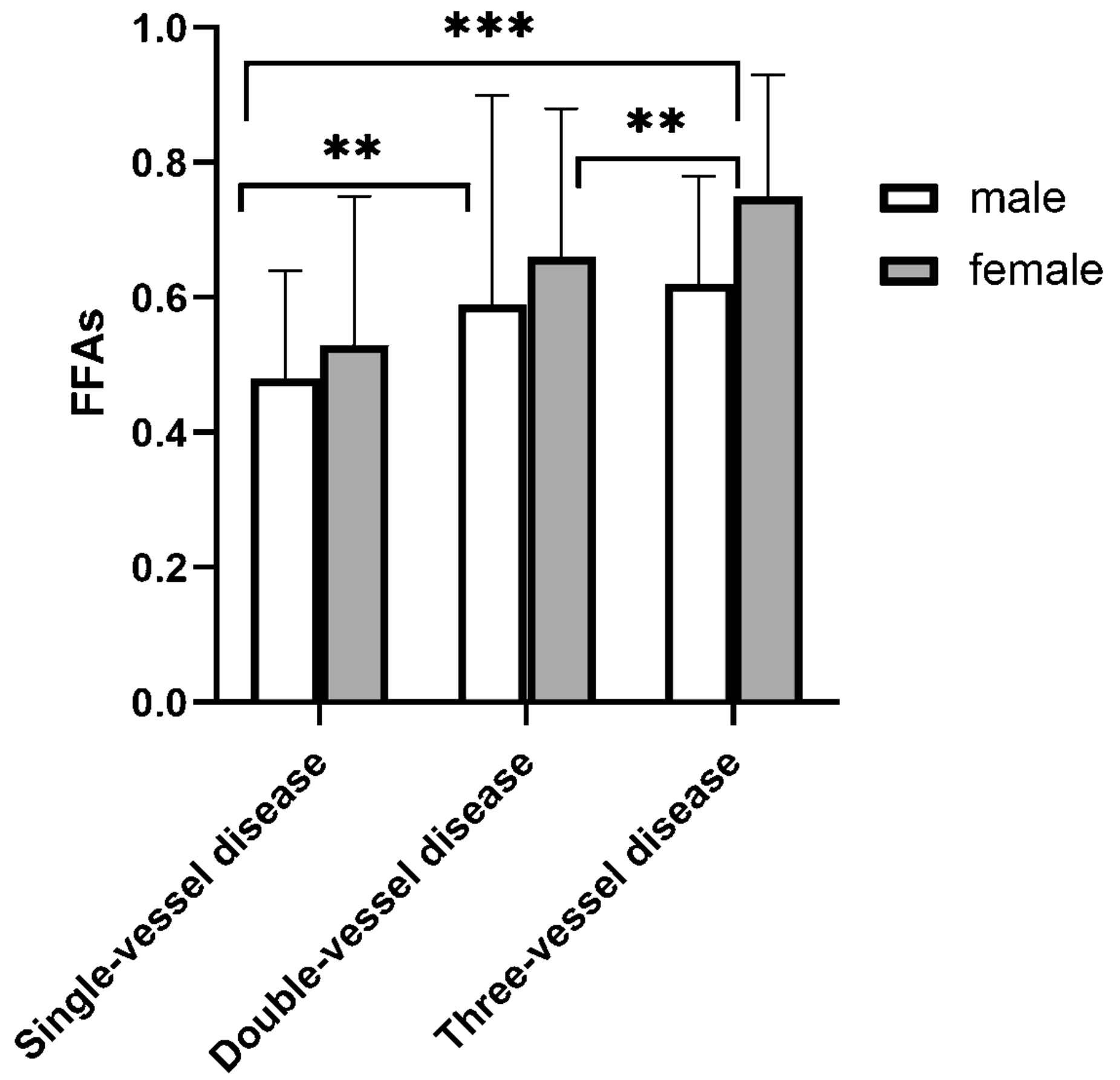

Figure 2

FFAs level in different genders based on complexity of CAD 


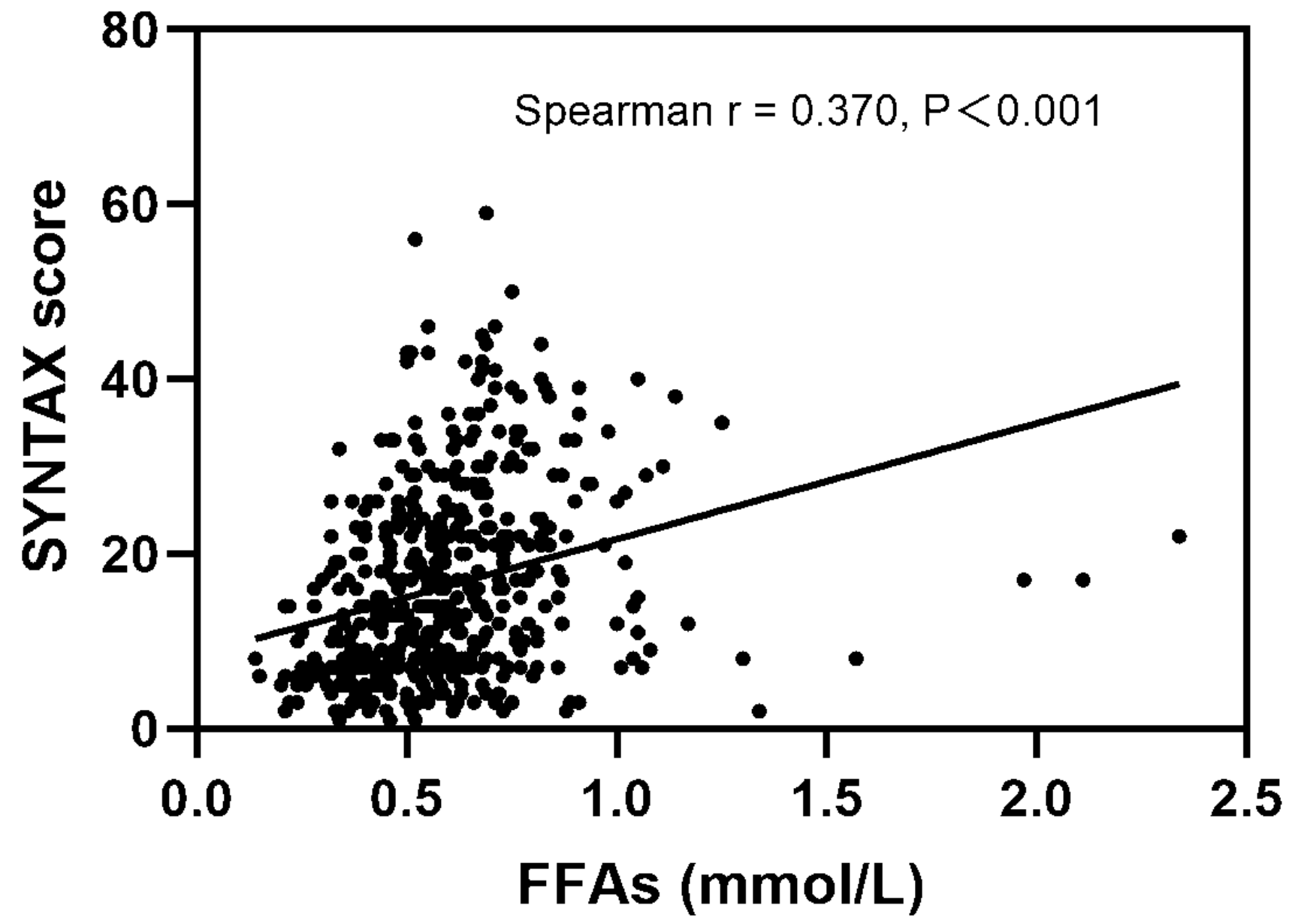

Figure 3

Spearman correlation analysis of FFAs level with SYNTAX score
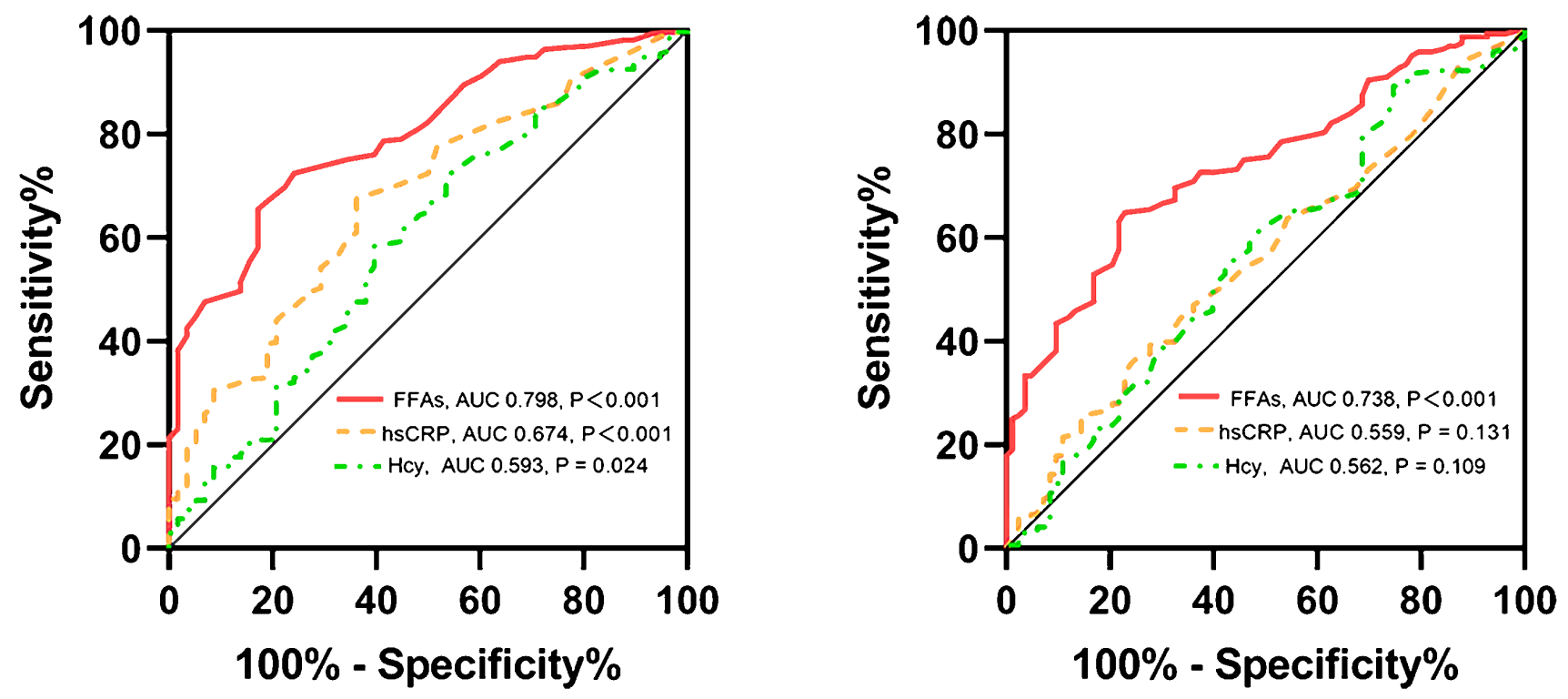
Figure 4

ROC curves of different indicators for diagnosing ACS in male patients (left) and female patients (right). FFAs: free fatty acids, hsCRP: high-sensitivity C-reactive protein, Hcy: homocysteine.

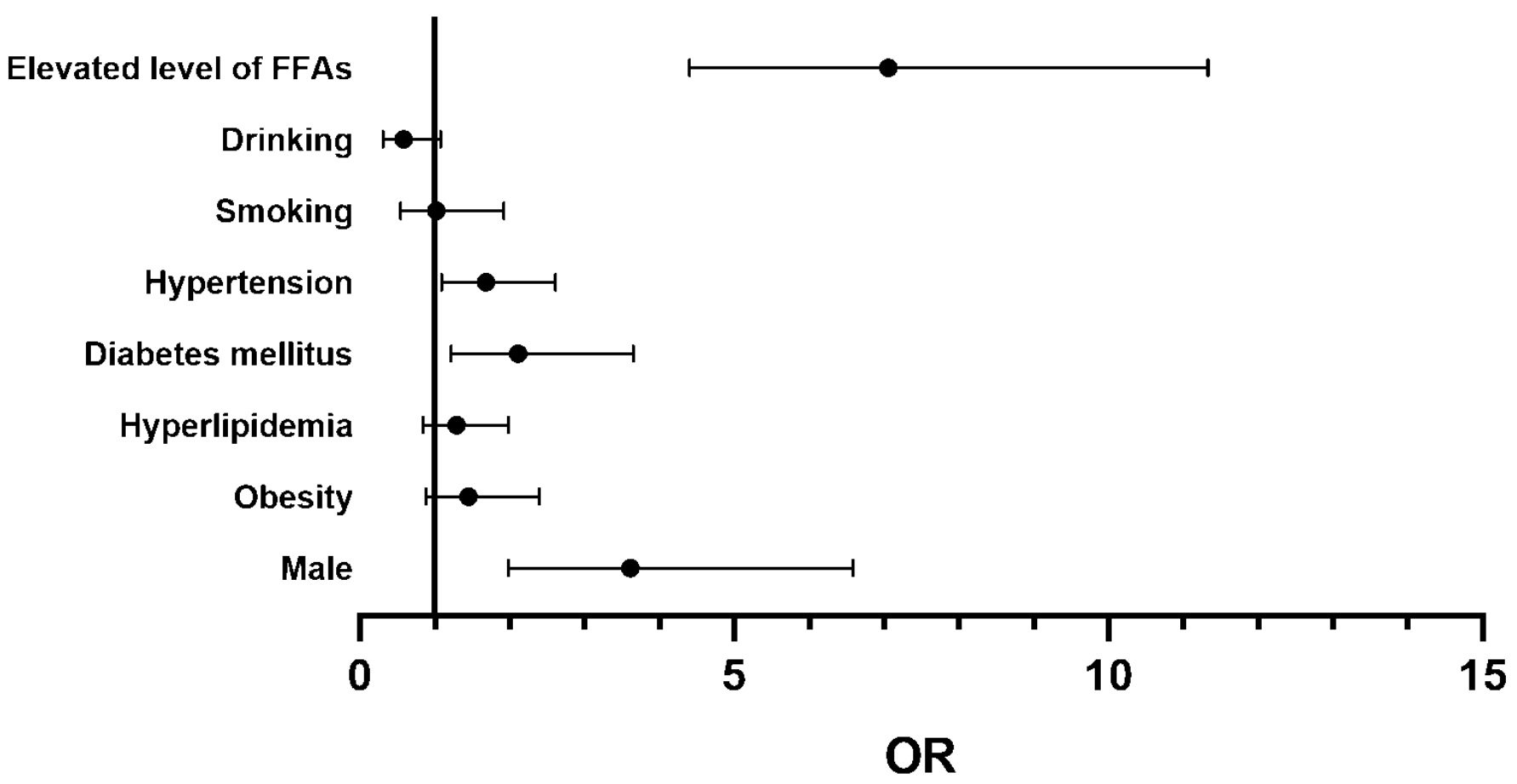

Figure 5

Forest plot of Multivariate logistic analysis of ACS risk factors 